Volume 9, No.3, May - June 2020

International Journal of Advanced Trends in Computer Science and Engineering

Available Online at http://www.warse.org/IJATCSE/static/pdf/file/ijatcse129932020.pdf

https://doi.org/10.30534/ijatcse/2020/129932020

\title{
Toward a Semantic Graph of Scientific Publications: A Bibliometric Study
}

\author{
Sara Mifrah ${ }^{1}$, El Habib Benlahmer ${ }^{2}$, Youssef Mifrah ${ }^{3}$, Mohamed Ezeouati $^{4}$ \\ ${ }^{1}$ Laboratory of Information Processing and Modeling, Hassan II University of Casablanca, Faculty of Sciences \\ Ben M'sik, Casablanca, Morocco, mifrah.sara@gmail.com \\ ${ }^{2}$ Laboratory of Information Processing and Modeling, Hassan II University of Casablanca, Faculty of Sciences \\ Ben Msik Casablanca, Morocco, h.benlahmer@gmail.com \\ ${ }^{3}$ Department of Software Engineering, National Institute of Posts and Telecommunications, INPT, Rabat, \\ Morocco, mifrah.youssef@gmail.com \\ ${ }^{4}$ Laboratory of Information Processing and Modeling, Hassan II University of Casablanca, Faculty of Sciences \\ Ben Msik Casablanca, Morocco, mohamed.ezeouati@gmail.com
}

\begin{abstract}
The term "scientific publication" includes several types of scientific communications and digital broadcasts that scientific researchers make of their work towards their peers and an audience of specialists. These publications describe in detail the studies or experiments carried out and the conclusions drawn from them by the authors. They undergo an examination of the value of the results and the rigor of the scientific method used for the work carried out. In this paper we evaluated the quality of a scientific article on the subject (topic), based on its citations and where is it cited, we based on the Topic modeling theme with the choice of LDA algorithms applied to the corpus Nips (1987-2016) for detecting all subjects of each paper and there citations in a first step then on the citations of each article of the corpus and on the Sentiment Analysis using a lexical based approaches. Then we created a csv file containing the link of each paper with the other cited papers (relation cited-citing), and finally generated a semantic graph between these publications.
\end{abstract}

Key words: Scientific publication, Citation analysis, Bibliometric, Citation graph, Semantic graph, Natural language Processing.

\section{INTRODUCTION}

When we say that an article is relevant, are we talking about the quantity of citations or the quality of the content?

This topic is the subject of recurrent debates while the number of scientific articles and the number of articles "cited" have increased sharply for two centuries, and even more for two decades due in particular to the appearance of the Internet and its search engines. Despite the establishment of scientometrics, bibliometrics [1] and management of scientific publication, authors [2][3][4][5][6][7] warn of the existence of misleading, biased or poor quality studies which pollute the research process and discredit science.
The criteria and procedures for evaluation are very much identical to those applied in the evaluation of research in general: peer reviews, panels, bibliometric indices, etc. The practical application of these tools in the case of development research may sometimes be quite difficult. Yet there seems to exist a general consensus that quality must be expressed in the same manner as for any scientific research, with the same problems and the same constraints.

The measurement of the quality of scientific productivity is problematic, no matter how quality is defined. In the case of publications, the most common method is to ask others in the same field to express their professional opinions. Obviously, the reliability of such assessments depend on the knowledge, objectivity and integrity of the assessors. What one person describes as a great contribution may be assessed as only modest by another or even regarded as contributing nothing by a third person. The peer review system is only as good as the people chosen to represent the peer group. The peer review process can sometimes be cumbersome because of difficulties in identifying appropriate peers, getting them to respond in reasonable time, and reconciling contrasting views when these arise. These are some of the reasons for the search for alternative measures of quality.

Bibliometric is a method of analyzing research activity, based on articles published in specialized scientific journals ("publications") [8]. These articles represent, in many sectors of academic research, an essential part of laboratory production. Bibliometric aims to establish relevant indicators to describe this production, and contribute to the analysis of research activity carried out in these laboratories [9]. Born on an empirical basis, difficult to handle, bibliometric-type indicators are nevertheless important tools for describing one of the essential dimensions of fundamental research.

This paper is structured as follows: Section 2 presents our research methodology. Section 3 the obtained results and finally, the conclusions of this paper are provided in Section. 4. 


\section{MATERIALS AND METHODS}

In order to assess the quality of a scientific publication (or report), we should distinguish between an intrinsic evaluation and an evaluation on the basis of external indicators. An intrinsic evaluation by an independent expert in the domain treated by the publication is generally considered to be the best way for assessing the scientific value of a paper.

Our evaluation approach based on the evaluation of the quality of the content of a scientific article, we added two other criteria: (1) the subject treated by the citing article and its relationship with the other cited (Topic Modeling) [10] and (2) the purpose of the citation (Sentiment Analysis).

\subsection{Data Set}

In this study we have used the Corpus NIPS (1987-2017) -Neural Information Processing Systems (NIPS) is one of the top machine learning conferences in the world. It covers topics ranging from deep learning and computer vision to cognitive science and reinforcement learning- This dataset includes the Id, year of publication, title, and extracted text for all NIPS papers to date (ranging from the first 1987 conference to the 2017 conference). We have extracted the paper text from the raw PDF files and are releasing that both in CSV files.

\subsection{Pre-processing}

In this phase we built a second csv file containing the citations of all papers in the corpus (Table 1), and a third one contains the references of each paper and the relationship "referenced by".

For the first part, we want to extract the citations from each paper, our input is a "file.txt" is the text content of an "article.pdf", we have segmented the text into sentences with delimiter (Uppercase-point), then we let's do a search on the sentences extracted previously, if they contain a number between brackets (exp. [x] VANCOUVER Style) if "yes", we consider this sentence as a "citation".

For the construction of the third csv file we used the GROBID machine learning reference extraction library, this library has several functionalities, we used only one, it is the parsing of references and the extraction of meta-data from each (authors, paper title, journal or conference, ISSN, publication date... ), and for the construction of the relation referenced by we find if a reference of an article exists in our corpus, if "yes" we trace this relation, if "no" we find elsewhere then we trace the relation with the "URL" where the article exists.

\subsection{Processing and analysis}

In the field of information analysis and information retrieval, we are interested to analyzed citations and extracted [18] their relationship with referenced papers. We are started with the detection of the context of citation; either in a sentence or for more precision within a paragraph. For example, we can take sentences from the first one containing the citation to the following sentence containing the other citation or to the end of the paragraph. This step call in the field of "Information Retrieval" by "segmentation". It consists of identifying the sentences in a sequence of words, we are only interested in sentences containing references, in this way we extracts each sequence of words containing one or more references, we obtain afterwards a list of sentences as output.

For detecting the semantic links and Topic Modeling, a second preprocessing step - applied on the previous extracted sentences (citations) - is necessary before processing algorithms. This step named in Information Retrieval System by "Lemmatization": One of the most important treatments in text mining applications, is the processing of the natural

Table 1: Example of citations

\begin{tabular}{|l|l|l|l|}
\hline ID paper & Year & Title & Citations \\
\hline 2 & $\mathbf{1 9 8 7}$ & $\begin{array}{l}\text { The Capacity of the } \\
\text { Kanerva Associative } \\
\text { Memory is Exponential }\end{array}$ & $\begin{array}{l}\text { This exponential grovth in capacity for the Kanerva associative memory contrasts } \\
\text { sharply yith the sub-linear grovth in capacity for the Hopfield associative memory [1] }\end{array}$ \\
\hline 6716 & $\mathbf{1 9 8 8}$ & $\begin{array}{l}\text { Adaptive Neural Networks } \\
\text { Using MOS Charge } \\
\text { Storage }\end{array}$ & $\begin{array}{l}\text { DAC's have already proven themselves in situations where 5 bits or less of } \\
\text { resolution [3] [4] are sufficient, but higher resolution is prohibitively expensive in } \\
\text { terms of area }\end{array}$ \\
\hline 7189 & $\mathbf{2 0 1 7}$ & $\begin{array}{l}\text { Improving Regret Bounds } \\
\text { for Combinatorial } \\
\text { Semi-Bandits with } \\
\text { Probabilistically Triggered } \\
\text { Arms and Its Applications }\end{array}$ & $\begin{array}{l}\text { Here we briefly explain the novel aspects of our analysis that allow us to achieve } \\
\text { new regret bounds and differentiate us from previous analyses such as the ones in } \\
\text { [7] and [16, 15] }\end{array}$ \\
\hline $\mathbf{2 0 1 7}$ & $\begin{array}{l}\text { Convolutional Phase } \\
\text { Retrieval }\end{array}$ & $\begin{array}{l}\text { The authors in [5, 2] show that the phase retrieval problem with random coded } \\
\text { distraction and STFT measurements can be solved by minimizing nonconvex } \\
\text { objectives, while [5] requires resampling for the initialization, and in [2] the } \\
\text { contraction radius is not large enough for initialization }\end{array}$ \\
\hline
\end{tabular}


language. This task is highly dependent on the language being processed, it can be divided into two steps: first one is to build a list of empty words that do not share a particular meaning useful for finding information for each language (elimination of stop words), and the second is the lexical analysis of the content of a text grouping the words of the same family.

\subsubsection{Text sentiment extraction}

In this step of sentiment analysis [19] we are used the Lexical dictionary WordNet and we have worked with the three classical classes (neutral, positive, negative) (Figure1).

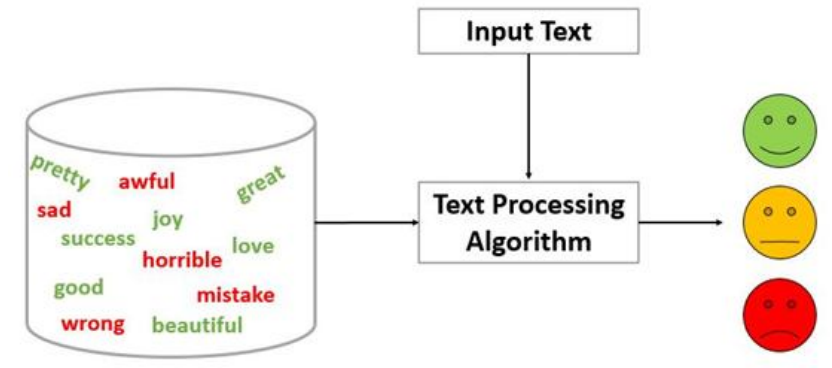

Figure 1: Polarity Extraction Processes of words which are semantically related. For that purpose it uses a statistical measure called Mutual Information, or MI for short. The higher the MI for a given pair of words, the higher the chance that they are related.

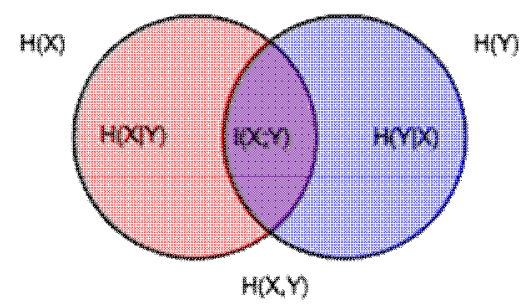

Figure 2: Venn diagram

Venn diagram [11] showing additive and subtractive relationships various information measures associated with correlated variables $\mathrm{X}$ and $\mathrm{Y}$. The area contained by both circles is the joint entropy $\mathrm{H}(\mathrm{X}, \mathrm{Y})$ (Figure 2). The circle on the left (red and violet) is the individual entropy $\mathrm{H}(\mathrm{X})$, with the red being the conditional entropy $\mathrm{H}(\mathrm{X} \mid \mathrm{Y})$. The circle on the right (blue and violet) is $\mathrm{H}(\mathrm{Y})$, with the blue being $\mathrm{H}(\mathrm{Y} \mid \mathrm{X})$. The violet is the Mutual Information $\mathrm{I}(\mathrm{X} ; \mathrm{Y})$

\subsubsection{Identification of semantic links}

Semantic Link analyzes the text and attempts to find all pairs

Table 1: Polarity of Citations

\begin{tabular}{|l|l|l|l|l|}
\hline $\begin{array}{c}\text { ID } \\
\text { paper }\end{array}$ & Year & Title & Citations & Polarity \\
\hline 2 & $\mathbf{1 9 8 7}$ & $\begin{array}{l}\text { The Capacity of the Kanerva } \\
\text { Associative Memory is } \\
\text { Exponential }\end{array}$ & $\begin{array}{l}\text { This exponential grovth in capacity for the Kanerva associative memory contrasts sharply yith the } \\
\text { sub-linear grovth in capacity for the Hopfield associative memory [1] }\end{array}$ & Neutral \\
\hline 110 & $\mathbf{1 9 8 8}$ & $\begin{array}{l}\text { Adaptive Neural Networks } \\
\text { Using MOS Charge Storage }\end{array}$ & $\begin{array}{l}\text { DAC's have already proven themselves in situations where 5 bits or less of resolution [3] [4] are } \\
\text { sufficient, but higher resolution is prohibitively expensive in terms of area }\end{array}$ & Negative \\
\hline 6716 & $\mathbf{2 0 1 7}$ & $\begin{array}{l}\text { Improving Regret Bounds for } \\
\text { Combinatorial Semi-Bandits } \\
\text { with Probabilistically } \\
\text { Triggered Arms and Its } \\
\text { Applications }\end{array}$ & $\begin{array}{l}\text { Here we briefly explain the novel aspects of our analysis that allow us to achieve new regret bounds } \\
\text { and differentiate us from previous analyses such as the ones in [7] and [16, 15] }\end{array}$ & Positive \\
\hline 7189 & $\mathbf{2 0 1 7}$ & $\begin{array}{l}\text { Convolutional Phase } \\
\text { Retrieval }\end{array}$ & $\begin{array}{l}\text { The authors in [5, 2] show that the phase retrieval problem with random coded distraction and } \\
\text { STFT measurements can be solved by minimizing nonconvex objectives, while [5] requires } \\
\text { resampling for the initialization, and in [2] the contraction radius is not large enough for } \\
\text { initialization }\end{array}$ & Negative \\
\hline
\end{tabular}

\subsubsection{Topic Model}

In this stage we have using the Latent Dirichlet Allocation (LDA) algorithm to detect the subjects of the scientific document and these citations, LDA [12] [13], is a probabilistic thematic modeling algorithm. It is based on the intuition that documents are composed of several themes (not words), where a theme is a multinomial distribution on a fixed vocabulary W [14]. 
The generative process for each document thus declines [15]:

1. Choose $\mathrm{N} \sim$ Poisson $(\xi)$.

2. Choose $\theta \sim \operatorname{Dir}(\alpha)$.

3. For each $\mathrm{N}$ words $w n$ :

a) Choose a topic $z n \sim$ Multinomial $(\theta)$.

b) Choose a word $w n$ from $\mathrm{p}(w n \mid z n, \beta)$, A conditioned multinomial probability on the subject zn

Where:

- $\xi$ : Random variable generating the number of words for each document.

- $\alpha$ : Vector of dimension K corresponding to the parameters of the Dirichlet law generating the words of a document.
- $\beta$ : The coefficients $\beta i j$ thus correspond to the probability of a word $\mathrm{w} j$ belonging to the topic $\mathrm{z} i$, thus $\beta i j=P(\mathrm{w} j \mathrm{z} i)$.

- $\theta$ : Latent variable represents the exact proportion of topics in each document.

- z: ziAre the topics (classes) associated with each word $w j$ of a document.

LDA sees each document as a set of occurrences appearing in an arbitrary order, which brings it, closer to the "bag-of-words" model [16].

Table 2: Extracted topics from citations

\begin{tabular}{|c|c|c|c|c|}
\hline $\begin{array}{c}\text { ID } \\
\text { Paper }\end{array}$ & Year & Title & Citations & Score-Topic \\
\hline 2 & 1987 & $\begin{array}{l}\text { The Capacity } \\
\text { of the } \\
\text { Kanexus As- } \\
\text { sociative } \\
\text { Memory is } \\
\text { Exponential }\end{array}$ & $\begin{array}{l}\text { This exponential grovth in } \\
\text { capacity for the Kanerva } \\
\text { associative memory con- } \\
\text { trasts sharply yith the sub- } \\
\text { linear grovth in capacity for } \\
\text { the Hopfield associative } \\
\text { memory [1]. }\end{array}$ & 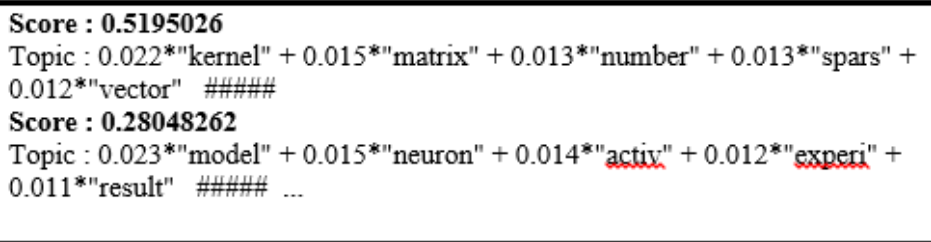 \\
\hline 110 & 1988 & $\begin{array}{l}\text { Adaptive } \\
\text { Neural Net- } \\
\text { works Using } \\
\text { MOS Charge } \\
\text { Storage }\end{array}$ & $\begin{array}{l}\text { DAC's have already proven } \\
\text { themselves in situations } \\
\text { where } 5 \text { bits or less of reso- } \\
\text { lution [3] [4] are sufficient, } \\
\text { but higher resolution is pro- } \\
\text { hibitively expensive in } \\
\text { terms of area. }\end{array}$ & 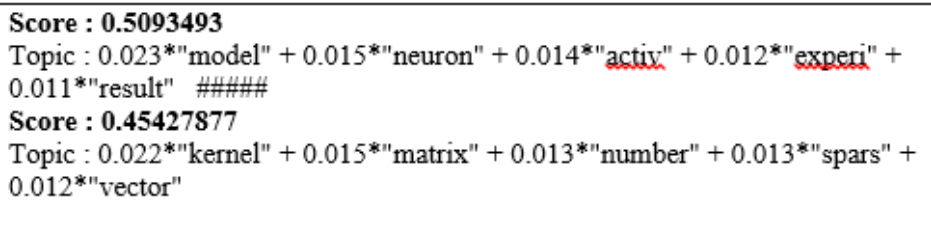 \\
\hline 7189 & 2017 & $\begin{array}{l}\text { Convolutional } \\
\text { Phase Re- } \\
\text { trieval }\end{array}$ & $\begin{array}{l}\text { The authors in }[5,2] \text { show } \\
\text { that the phase retrieval } \\
\text { problem with random } \\
\text { coded difraction and STFT } \\
\text { measurements can be } \\
\text { solved by minimizing non- } \\
\text { convex objectives, while } \\
\text { [5] requires resampling for } \\
\text { the initialization, and in [2] } \\
\text { the contraction radius is } \\
\text { not large enough for ini- } \\
\text { tialization. }\end{array}$ & $\begin{array}{l}\text { Score : } \mathbf{0 . 4 4 5 2 5 7 9 6} \\
\text { Topic : } 0.029^{*} \text { result" }+0.027^{*} \text { "follow" }+0.025^{* 1 "} \text { theorem" }+0.021^{*} \text { "function" } \\
+0.020^{*} \text { "graph" \#\#\#\# } \\
\text { Score }: \mathbf{0 . 4 4 0 4 4 4} \\
\text { Topic }: 0.043^{*} \text { "algorithm" }+0.033^{*} \text { "problem" + } 0.032^{*} \text { "optim" + } 0.018^{*} \text { "gradi- } \\
\text { ent" }+0.015^{*} \text { "method" \#\#\#\# ... }\end{array}$ \\
\hline
\end{tabular}

\subsubsection{Topic visualization}

Though we've already seen what the topic keywords in each topic are, a word cloud with the size of the words proportional to the weight is a pleasant sight. The coloring of the topics we've taken here is followed in the subsequent plots as well 


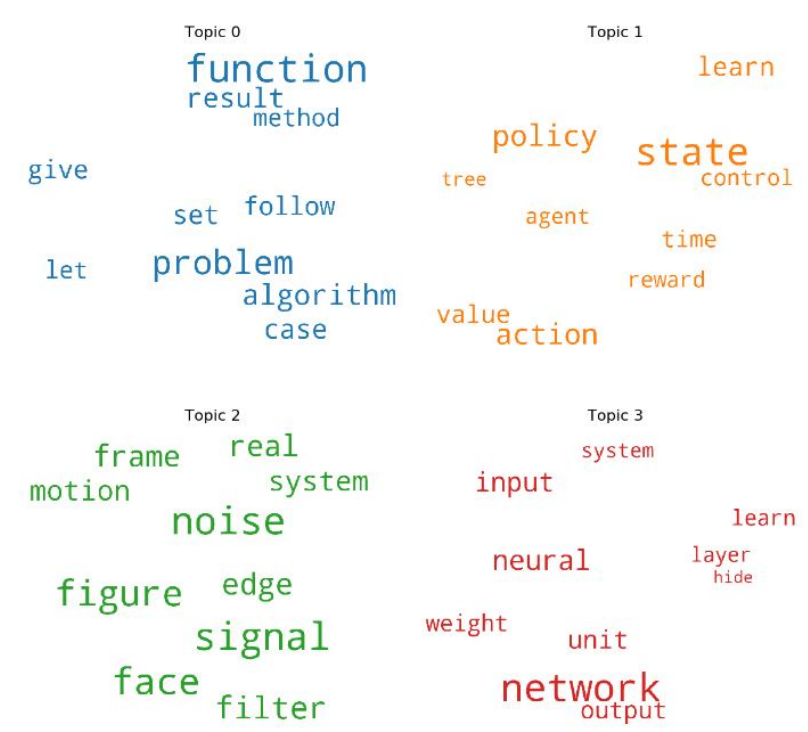

When it comes to the keywords in the topics, the importance (weights) of the keywords matters. Along with that, how frequently the words have appeared in the documents is also interesting to look.

We want to keep an eye out on the words (Figure 3) that occur in multiple topics and the ones whose relative frequency is more than the weight. Often such words turn out to be less important. The chart we've drawn below is a result of adding several such words to the stop words list in the beginning and re-running the training process.
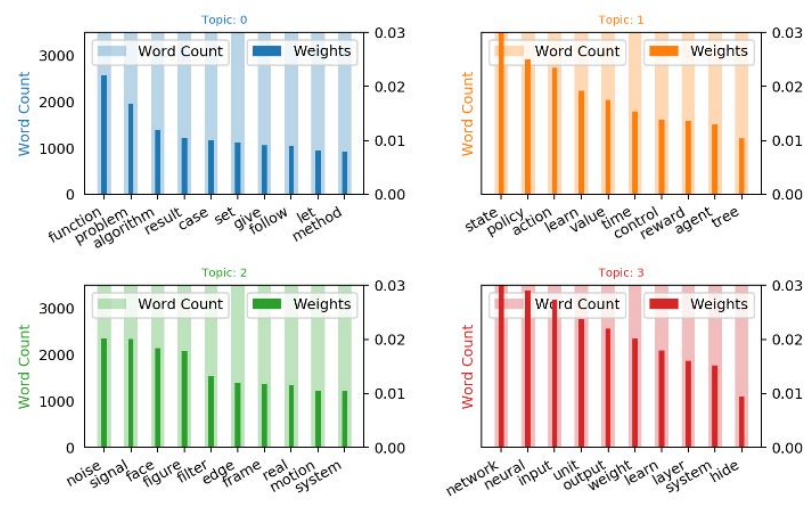

Figure 3: Word Count and Importance of Topic Keywords

\subsubsection{Citing-Cited relationship}

To create this tree structure (Figure 4), we created a reference relationship between all articles in the corpus (Table 3); as a starting point, we used Grobid1 (GeneRation Of BIbliographi--c Data)

\footnotetext{
${ }^{1}$ A machine learning software for extracting information from scholarly documents https://grobid.readthedocs.io
}

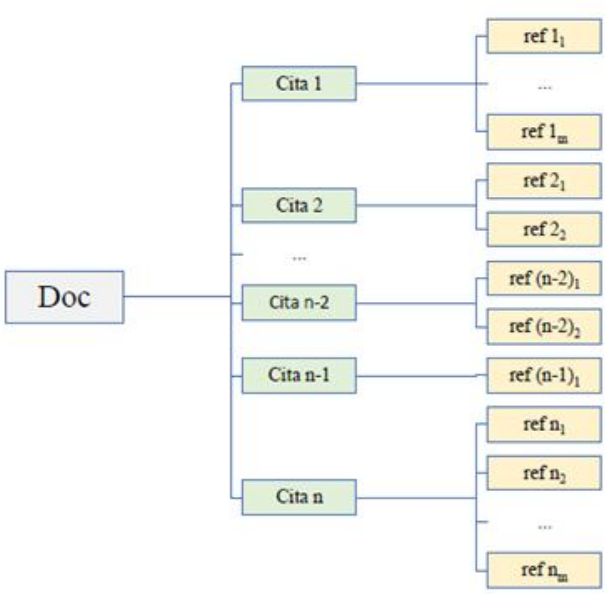

Figure 4: Relational schema of a scientific document with "ref" is a document

for extracting the references for each paper in the corpus in PDF format as input, and output as structured XML/TEI encoded documents. By the following we created a program takes as input an XML / TEI document to make parsing then extracts the titles of the references, find if the titles extracted exists in our corpus, as output we created a file csv with the form (id; pdf-name ; references; referenced by; title; pub-year).

The referenced by or cited relationship allows us to establish a relationship between the articles in the same corpus.

After our experimentation on NIPS corpus we observed that only the articles in the period (1987-1997) which change this relation, the number of these articles is more than 200 articles among 3543 between the periods (1987-2016) (Table 4). 
Table 3 : "Referenced by" relationship or Cited

\begin{tabular}{|c|c|c|c|c|}
\hline ID & PDF_NAME & REFRENCED_BY & TITLE & Pub_YEAR \\
\hline 1001 & |1001-neural-network-ensembles-cross-vi & i. Information, prediction, and query by committee $\gg \mathrm{Bi}$ Generating Accurate and Divorse Member: & : Neural NetworkEnsemilote, Crossvalidation, and Active Le: & 1994 \\
\hline 1000 & 1000-bayesian-query-construction-for-n & >>Information-Based Objective Functions for Active [active Learnin & Bayesian Query C & 1994 \\
\hline 1009 & 1009-an-experimental-comparison-of-re & FIR and IIR synapses, a new neural network architecture Learminglong-term dependoneiesis not as & s An experimental comparison of recurront newaitiletworks & 1994 \\
\hline 1010 & 1010-interference-in-learning-internal-n & Two problems with backpropagation and other steepes Catastrophic Interference in Human Moto & Interference intearning internel Models of Inverse Dynami & 1994 \\
\hline 1011 & 1011-active-learning-with-statistical-mo & 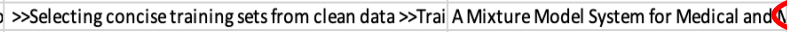 & Active Learning with Statistical Models & 1994 \\
\hline 1013 & 1013-ocular-dominance-and-patterned-1 & Ocular dominance column development: Strabismus ch Laterally Interconnected Self-Organizing N & Vcular Dominancoand Petterned Lateral Connections in a: & 1994 \\
\hline 1018 & 1018-generalization-in-reinforcement-le & Practical issues in temporal difference learning $\gg$ Autor Learning to Play the Game of Chess $\gg \gg$ & Generalization in Reinforcement Learning: Safely Approxim & 1994 \\
\hline 1019 & 1019-a-mixture-model-system-for-medic & ( Mixture Models: Inference and Applications to Clusterit Learning Decision Theoretic Utilities & A Mixture Model System for Medical and Mach & 1994 \\
\hline 102 & 102-an-application-of-the-principle-of-n & and Relial Deri & & 1988 \\
\hline 1022 & 1022-a-multiscale-attentional-framewor & euronal' Networks in Early Vision >>Mul & & 1995 \\
\hline 1028 & 1028-exponentially-many-local-minima. & - Neural Networks: a Comprehensive Foundation 〉>Back| Exponentially many local minima for singl & Exponentially many local minima for single neurons & 1995 \\
\hline 1029 & 1029-a-practical-monte-carlo-implemen & Regression with Gaussian processes 〉>Hybrid Monte Ca Gaussian Processes for Regression & APractical Monte Carlo Implementation of Bayesian Learni & 1995 \\
\hline 1036 & 1036-improved-gaussian-mixture-densit & Operations for learning with graphical models $\gg$ Estima Stacked Density Estimation & Improved Gaussian Mixture Density Estimates Using Bayesi: & 1995 \\
\hline 1041 & 1041-the-geometry-of-eye-rotations-and & Analysis, Manifolds and Physics $\gg$ Commun. Math. Phy A Dynamical Model of Context Depe & c The Geometry of Eye Rotations and Listing's Law & 1995 \\
\hline 1042 & 1042-reinforcement-learning-by-probab & Learning automata algorithms for connectionist system MIMIC: Finding Optima by Estimating Prol & Reinforcement Learning by Probability & 1995 \\
\hline 1048 & 1048-gaussian-processes-for-regression. $\mathrm{f}$ & f Networks for approximation and learning >>Regularizat Gaussian Processes for Bayesian Classificat & t Gaussian Processes for Regression & 1995 \\
\hline 1051 & 1051-neural-networks-with-quadratic-vi & ( Sigmoids distinguish better than Heavisides 〉>Perspect Size of Multilayer Networks for Exact Learr & r Neural Networks with Quadratic VCD & 1995 \\
\hline 1059 & 1059-boosting-decision-trees.pdf & >Memory-based approaches to approximating contin ARC-LH: A New Adaptive Resampling Algor & r Boosting Decision Trees & 1995 \\
\hline 1066 & 1066-on-neural-networks-with-minimal & 1. Learning in threshold networks: a computational mode Multiple Threshold Neural Logic & On Neural Networks with Minimal Weights & 1995 \\
\hline 107 & 107-neural-network-recognizer-for-hand & Some Studies in the Interactive Design of Character Rec Contour-Map Encoding of Shape for Early & ' Neural Network Recognizer for Hand-Written Zip Code Digi & 1988 \\
\hline 1073 & 1073-improving-elevator-performance-ı & 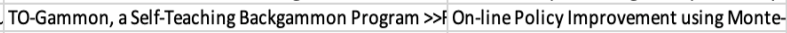 & - Improving Elevator Performance Using Reinforcement Lear & 1995 \\
\hline 1075 & 1075-improving-committee-diagnosis-w & V Stacked Regressions $\gg$ An Introd. to the Bootstrap $\gg$ St: Competition Among Networks Improves C & CImproving Committee Diagnosis with Resampling Techniqı & 1995 \\
\hline 108 & 108-neural-networks-for-model-matchir & r >Optimization in Model Matching and Perceptual Org Analog Circuits for Constrained Optimizat & Neural Networks for Model Matching and Perceptual Orgar & 1988 \\
\hline 1089 & 1089-beating-a-defender-in-robotic-socc & ( Pinball: Planning and learning in a dynamic real-time er Beating a Defender in Robotic Soccer: Men & n Beating a Defender in Robotic Soccer: Memory-Based Learn & 1995 \\
\hline 1095 & 1095-worst-case-loss-bounds-for-single-1 & 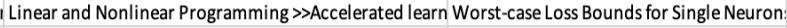 & Worst-case Loss Bounds for Single Neurons & 1995 \\
\hline 1098 & 1098-discovering-structure-in-continuo & Operations for learning with graphical models >>Probal Continuous Sigmoidal Belief Networks Tra & a Discovering Structure in Continuous Variables Using Bayesi & 1995 \\
\hline 1102 & 1102-hierarchical-recurrent-neural-netu & a Learning internal representations by error propagation Learning long-term dependencies is not as & s Hierarchical Recurrent Neural Networks for Long-Term Dep & 1995 \\
\hline 1111 & 1111-fast-learning-by-bounding-likeliho & Mean field theory for sigmoid belief networks. M.I. T. C. A Hierarchical Model of Visual Rival ry $\gg \mathrm{Cc}$ & ic Fast Learning by Bounding Likelihoods in Sigmoid Type Beli & 1995 \\
\hline 114 & 114-implications-of-recursive-distribute & How much do people remember? Some estimates on th Higher Order Recurrent Networks and Gra & Implications of Recursive Distributed Representations & 1988 \\
\hline 1144 & 1144-factorial-hidden-markov-models.p & Autoencoders, minimum description length, and Helm Hidden Markov Decision Trees & Factorial Hidden Markov Models & 1995 \\
\hline 115 & 115-heterogeneous-neural-networks-for & Insect walking $\gg$ The American Cockroach $\gg$ The contro Neural Implementation of Motivated Beha & : Heterogeneous Neural Networks for Adaptive Behavior in D & 1988 \\
\hline 1153 & 1153-does-the-wake-sleep-algorithm-prc & c Maximum likelihood from incomplete data via the EM c Estimating Dependency Structure as a Hid & Does the Wake-sleep Algorithm Produce Good Density Estiı & 1995 \\
\hline 1154 & 1154-control-of-selective-visual-attentic & c Features and objects: the fourteenth Bartlett memorial Correlates of Attention in a Model of Dyna & a Control of Selective Visual Attention: Modeling the "Where & 1995 \\
\hline 1155 & 1155-exploiting-tractable-substructures & s The Gibbs machine applied to hidden Markov model pr. Factorial Hidden Markov Models >>Appros & 1) Exploiting Tractable Substructures in Intractable Networks & 1995 \\
\hline 1158 & 1158-on-the-computational-power-of-n & On the computational complexity of networks of spikin Noisy Spiking Neurons with Temporal Cod & On the Computational Power of Noisy Spiking Neurons & 1995 \\
\hline
\end{tabular}

\section{RESULTS AND DISCUSSION}

Each citation in a scientific paper is linked at least to one reference's article (Figure 4).

After we got the results, we can incorporate the concept of the semantic class. We can say for example that:

«The author of the basic document (Doc 3 ) criticizes the approach $\boldsymbol{x}$ cited of the article referenced (Doc 7), in the subject $\boldsymbol{z}$ (Topic 5 ) with the proportion $\mathbf{5 2 \%} \%$.

With : $\boldsymbol{p} \in[0,100[$

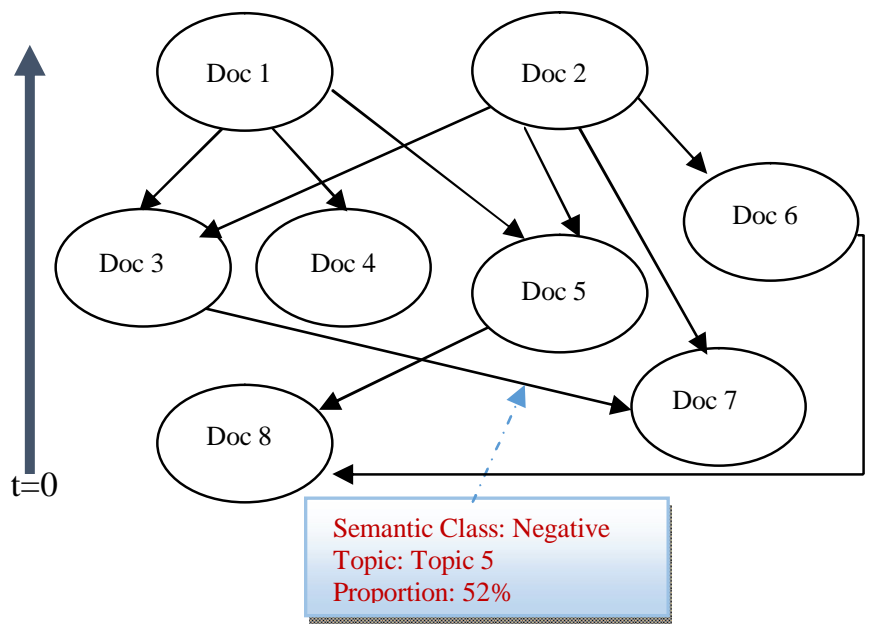

In order to get our result (Figure 6) we used the Graphvis tool, it is an open source graph visualization software. Graph visualization is a way of representing structural information as diagrams of abstract graphs and networks (Figure 5). It has important applications in networking, bioinformatics, software engineering, database and web design, machine learning, and in visual interfaces for other technical domains.

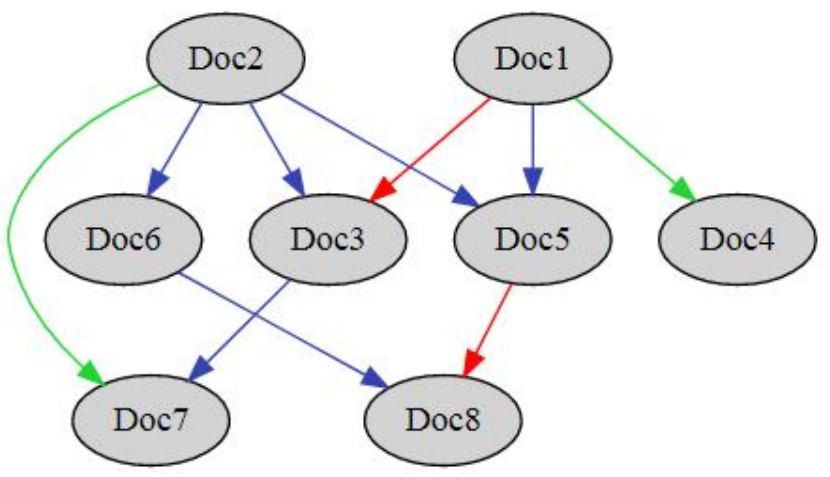

Figure 5: Generated Graph using Graphvis 


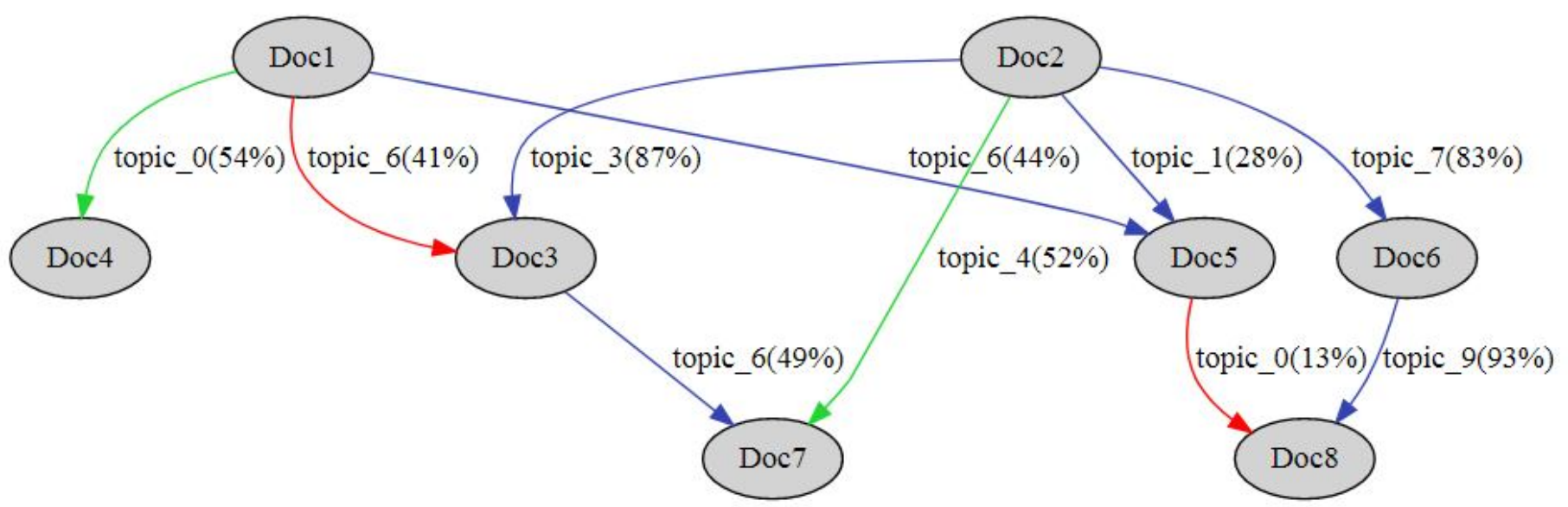

Figure 6: Example of relationship graph with topic \& semantic class

Each node in the graph represents a scientific article and each arc represents the reference or citation relation, moreover the color of the arc represents the semantic class and the label on each arc is the dominant topic of the citation.

To achieve our result we have generated a text file of the following form using Graphvis library:

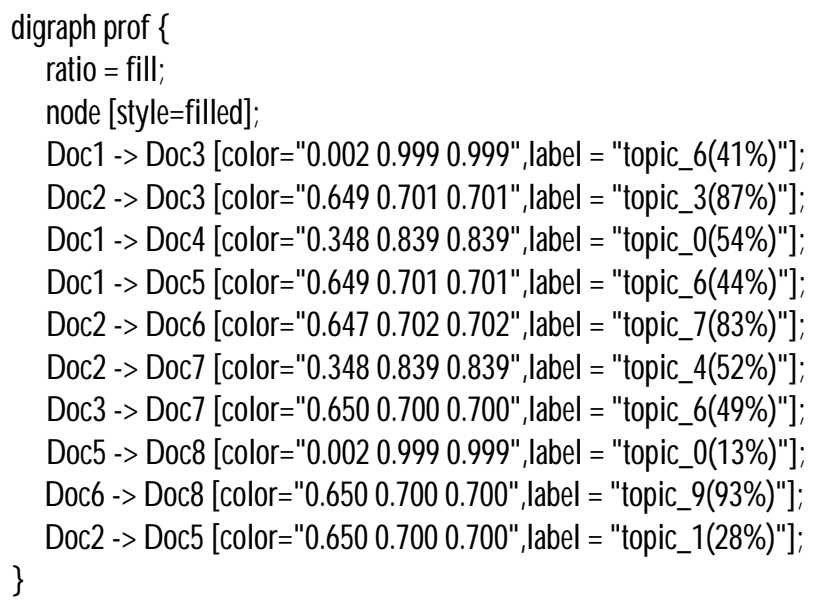

Each line represents the link of the two nodes; the color of the link arc and the label on the arc.

\section{CONCLUSION}

In conclusion, we can say that the analysis of scientific documents [17] is a topic that interests many people from different disciplines. We find, on the one hand, scientists (researchers) that produce and exploit these documents; on the other hand, scientific publishers, librarians, and producers of databases... who handle and process these scientific documents.

The better scientific articles you use in your literature review, the more robust your own research will be. If you use articles that are not authoritative, you run the risk of drawing unfounded conclusions harmful to your dissertation or thesis.
Your supervisor always checks the sources of the conclusions you draw.

\section{REFERENCES}

1. Weingart P. Impact of bibliometrics upon the science system: Inadvertent consequences? Scientometrics .62(1), 117-131. 2005

https://doi.org/10.1007/s11192-005-0007-7

2. Maddox J. Why the pressure to publish?. Nature 1988, 333, 493. https://doi.org/10.1038/333493a0

3. Angell, M. Publish or perish: a proposal. Annals of Internal Medicine, 104(2), 261-262. 1986 https://doi.org/10.7326/0003-4819-104-2-261

4. De Rond M \& Miller A.N. Publish or perish bane or boon of academic life? Journal of Management Inquiry, (2005) 14(4), 321-329. https://doi.org/10.1177/1056492605276850

5. Darnill A. Publish or perish. Accountancy, 118 (1,237), 1997.

6. Colquhoun D. Publish-or-perish: Peer review and the corruption of science .The Guardian, 5(09).

7. Lehrer J. The truth wears off: is there something wrong with the scientific method? The New Yorker.

8. Pritchard, A. Statistical Bibliography or Bibliometrics Journal of Documentation. 348-349. 1969

9. Vachon, Éric, Yves Petit, et Pierre Etève . Les indicateurs Bibliométriques en recherche. Éducation $\&$ formations, $n^{\circ} 59,123-128$, avril-juin 2001

10. Sara Mifrah and Ben Lahmar El Habib. Semantic Relationship Study between Citing and Cited Scientific Articles Using Topic Modeling. In Proceedings of the 4th International Conference on Big Data and Internet of Things (BDIoT'19), Rabat Morocco (2019). DOI: https://doi.org/10.1145/3372938.3372943

11. Venn Diagram [Availabale] https://en.wikipedia.org/wiki/Venn_diagram 
12. Blei, D., Ng, A., \& Jordan, M.. Latent dirichlet allocation. The Journal of Machine Learning Research 3, 993-1022.

13. 12 Griffiths, T. L., \& Steyvers, M. Finding scientific topics. doi: 10.1073/pnas.0307752101 PNAS April 6, 2004 vol. 101 no. suppl 1, 5228-5235. 2004 https://doi.org/10.1073/pnas.0307752101

14. 13 Deveaud, R., Bonnefoy, L., \& Bellot, P. Quantification et identification des concepts implicites d'une requête. [Availabale] http://coria.unine.ch/coria/coria2013_27.pdf.2013

15. Francesiaz, T., Graille, R., \& Metahri, B. Introduction aux modèles probabilistes utilisés en fouille de données. Retrieved juillet 20, 2016 [Availabale] http://www-ljk.imag.fr/membres/Marianne.Clausel/Fich iers/Rapport_Metahri_Graille_Francesiaz.pdf

16. Rigouste, L., Cappe, O., \& Yvon, F. Quelques observations sur le modèle LDA. 2006. [Availabale] https://perso.limsi.fr/yvon/publications/sources/Rigouste 06lda.pdf

17. Mifrah, S., Benlahmer, El H.: Semantico-automatic Evaluation of Scientific Papers: State of the Art. BDCA'17 Proceedings of the 2nd international Conference on Big Data, Cloud and Applications Tetouan, Morocco — March 29 - 30, 2017 ISBN: 978-1-4503-4852-2 DOI: 10.1145/3090354.3090380 (2017)

18. Kadu, Payal, and Ashwini V. Zadgaonkar. "Knowledge Extraction from Text Document Using Open Information Extraction Technique." International Journal of Advanced Trends in Computer Science and Engineering (IJATCSE) Volume 9, No.2 ISSN 2278-3091, DOI: 10.30534/ijatcse/2020/208922020 (2020).

19. Ali Muttaleb Hasan et al., "Knowledge-Based Semantic Relatedness measure using Semantic features" International Journal of Advanced Trends in Computer Science and Engineering (IJATCSE) Volume 9, No.2 ISSN 2278-3091 Volume 9 No.2, March - April 2020 DOI/ 10.30534/ijatcse/2020/02922020 